\title{
Assessment of Uterine Receptivity by the Subendometrial- Endometrial Blood Flow Distribution Pattern in Frozen- thawed Embryo Transfer Cycles
}

\author{
Divya Sardana
}

\begin{abstract}
Objective: To investigate the correlation of blood flow detected by 2-Dimensional Power Doppler (2D-PD) sonography in the subendometrial-endometrial unit with the pregnancy outcome in frozen-thawed embryo transfer (FET) cycles.
\end{abstract}

Study design: Prospective non-randomized observational study. Materials and methods: A total of 127 patients undergoing their first FET cycle were recruited and prepared using hormone replacement therapy. All patients were evaluated for subendometrial-endometrial blood flow by 2D-PD once the endometrium was $\geq 7 \mathrm{~mm}$ thick. Progesterone supplement was added and transfer of 2 to 3 cleavage stage good quality embryos was done after 3 days.

Results: The difference in the BMI, $\mathrm{FSH}, \mathrm{LH}$ and endometrial thickness of women in the three zones was not statistically significant $(p>0.05)$. However, women with blood flow in zone 1 were of older age as compared to women in zone 3 with a statistically significant difference $(p=0.0001)$. In the present study, the overall pregnancy rate was $35.43 \%$ (45/127). Women having blood flow in zone 3 or 4 showed a rising trend of pregnancy rate as compared to those in zone 1 and 2 although it was not statistically significant $(p=0.15)$. But, clinical pregnancy and implantation rates were significantly higher in women with zone 3 or 4 penetration compared with zone 1 or 2 penetration $(p=0.03$ and 0.01 , respectively).

Conclusion: The subendometrial-endometrial vascularity has a useful predictive value for pregnancy outcome in FET cycles. However, larger studies are needed to reach definite conclusion.

Keywords: Power doppler, Frozen-thawed embryo transfer, Subendometrial-endometrial unit.

How to cite this article: Sardana D. Assessment of Uterine Receptivity by the Subendometrial-Endometrial Blood Flow Distribution Pattern in Frozen-Thawed Embryo Transfer Cycles. Int J Infertil Fetal Med 2015;6(1):35-39.

Source of support: Nil.

Conflict of interest: None

Date of Received: $12-01-15$

Date of Acceptance: 25-02-15

Date of Publication: April 2015

\section{Fellow}

Department of Reproductive Medicine, BACC Healthcare Pvt Ltd, Bengaluru, Karnataka, India

Corresponding Author: Divya Sardana, Fellow, Department of Reproductive Medicine, BACC Healthcare Pvt Ltd Bengaluru, Karnataka, India, Phone: 22260880, e-mail: drdivyasardana80@gmail.com

\section{INTRODUCTION}

Inspite of various advancements in the field of assisted reproductive techniques (ART), pregnancy rates still remain low and endometrial receptivity remains its bottleneck. Ultrasound has emerged as a noninvasive and an indispensable modality for the assessment of endometrium. Endometrial thickness and pattern have been the most commonly used parameters to assess endometrial preparation. With the advent of Doppler and its various types, evaluation of endometrial blood flow also has gained importance in recent times.

Blastocyst implants into the endometrium and hence, evaluation of endometrial blood flow is more appropriate than uterine artery blood flow parameters. ${ }^{1,2}$ However, conflicting results have been reported about the assessment of blood flow in subendometrial-endometrial unit in predicting pregnancy outcome in in vitro fertilization (IVF) cycles..$^{3-9}$ While there is extensive literature on the correlation of endometrial blood flow and pregnancy outcome in IVF cycles, there is scarcity of data of the same in frozen-thawed embryo transfer (FET) cycles.

The aim of our study was to investigate the correlation of blood flow in the subendometrial-endometrial unit detected by 2-Dimensional Power Doppler (2D-PD) sonography with the pregnancy outcome in FET cycles.

\section{MATERIALS AND METHODS}

It was a prospective non-randomized observational study where 127 women undergoing their first frozen embryo transfer (FET) cycle in our infertility center from June 2011 to December 2012 were recruited. The study was approved by the Ethical Committee of the hospital, and all patients consented to participate in the study.

Inclusion criteria included women between 20 and 40 years of age, undergoing their first FET cycle, where vitrification technique was used for freezing and at least two or three good quality embryos were available for transfer after thawing. Patients with distorted uterine cavity on transvaginal ultrasound (TVS) or hysteroscopy, as well as with hydrosalpinx or fibroids were excluded from the study.

All patients underwent antagonist IVF cycle of stimulation and cleavage stage (6-8 cell) good quality embryos 
were frozen on day 3 of oocyte retrieval by vitrification technique when the embryos were surplus for fresh cycle or when fresh transfer was not possible due to risk of OHSS.

For the FET cycle (at least after 2 months of oocyte retrieval), the endometrium was prepared using hormone replacement therapy with estradiol and progesterone supplements. All patients underwent TVS to assess uterine and ovarian morphology on days 2 of spontaneous or induced menstrual cycle. Estradiol supplementation (Tab Estradiol valerate $2 \mathrm{mg}$ TID) was started on days 2 or 3 of menses once endometrial thickness was less than $6 \mathrm{~mm}$ and no ovarian cyst was seen on ultrasound. All patients also received folic acid supplements. Ultrasound was repeated after a week of hormone replacement therapy (HRT) and once endometrial thickness was $>7 \mathrm{~mm}$, Doppler study of endometrial blood flow was conducted in all patients.

All ultrasound scans and measurements were performed with the digital platform VOLUSON E8 system between 9 and 11 am. A B-Mode exploration of both uterus and ovaries was initially done. Then the endometrium was assessed in the longitudinal section for its thickness, which was measured from outside to outside at its widest point. Then 2D-PD assessment was done. The Power Doppler characteristics applied in all examinations were normal quality of color, color gain-3.4, pulse repetition frequency of $600 \mathrm{~Hz}$ and wall motion filter of $50 \mathrm{~Hz}$.

Using Power Doppler, the endometrial blood flow distribution pattern was determined by demonstrating color signals in the subendometrial-endometrial regions. For the zones of endometrial blood flow, we followed the definition by Applebaum, ${ }^{10}$ summarized as follows: zone 1 , vessels penetrating the outer $2 \mathrm{~mm}$ hypoechogenic area surrounding the endometrium but not entering the hyperechogenic outer margin; zone 2, vessels penetrating the hyperechogenic outer margin of the endometrium but not entering the hypoechogenic inner area; zone 3, vessels entering the hypoechogenic inner area; and zone 4, vessels entering the hyperchogenic cavity.

Progesterone supplement (Inj Progesterone $100 \mathrm{mg}$ i.m. for 3 days) was added and cleavage stage embryo transfer ( 2 or 3 good quality embryos) was done after
3 days. All patients were prescribed vaginal progesterone suppositories and estradiol valerate tablets for luteal phase support after embryo transfer along with folic acid supplements for 2 weeks after which blood was tested for $\beta$-hCG. $\beta$-hCG $>50 \mathrm{IU} / \mathrm{L}$ was considered as positive for pregnancy. Clinical pregnancy was defined by the appearance of gestational sac on ultrasound with appropriately rising serum $\beta$-hCG levels. When no gestational sac could be seen with positive $\beta$-hCG by 6 weeks, it was defined as a biochemical pregnancy.

The primary and secondary outcomes of the study were clinical pregnancy rate and implantation rate, respectively.

\section{STATISTICAL ANALYSIS}

In the present study, data were collected on SPSS-16 and Microsoft Excel Sheet 2010. Comparison of mean for Age, $\mathrm{BMI}, \mathrm{FSH}, \mathrm{LH}$ and endometrium across the three zones was done using ANNOVA. Bonferroni test was used for post-hoc analysis, and $\mathrm{p}$ value of $<0.05$ taken as statistically significant. Chi-square test was used to evaluate strength of association between Doppler findings and FET cycle outcomes, and $p$ value of $<0.05$ taken as statistically significant.

\section{RESULTS}

A total of 127 women were recruited in the study of which 19 women had blood flow in zone 1,37 women had blood flow in zone 2 and 71 patients had blood flow in zone 3 or 4 . Eighty two patients had primary infertility [64.56\% (82/127)] and 45 patients had secondary infertility $[35.43 \%$ (45/127)]. The difference in the BMI, FSH, LH and endometrial thickness of women in the three zones of blood flow was not statistically significant ( $p>0.05$ ). However, women with blood flow in zone 1 were of older age as compared to women in zone 3 with a statistically significant difference $(\mathrm{p}=0.0001)$ (Table 1$)$.

In the present study, the overall pregnancy rate was $35.43 \%$ (45/127). Women having blood flow in zone 3 or 4 penetration showed a rising trend of pregnancy rate [42.25\% (30/71)] as compared to those in zone 1 (21.05\% [4/19]) and zone 2 [29.72\% (11/37)] respectively, although it was not statistically significant $(\mathrm{p}=0.15)$. But, clinical

Table 1: Demographic, endometrial thickness data and hormonal profile of women in the three zones

\begin{tabular}{lllll}
\hline Mean & Zone1 ${ }^{* * *}(S D)$ & Zone2 $^{* * *}(S D)$ & Zone3 $^{* * *}(S D)$ & $€ p$-value (<0.05) \\
\hline Age in years & $34.95(4.23)$ & $32.54(3.92)$ & $31.03(3.37)$ & 0.0001 \\
${ }^{*}$ BMI in kg/m & $26.74(2.75)$ & $26.82(2.70)$ & $25.93(2.68)$ & 0.20 \\
${ }^{* *}$ ET in mm & $9.57(1.16)$ & $9.97(1.24)$ & $9.91(1.15)$ & 0.47 \\
†FSH (IU/L) & $5.42(1.46)$ & $6.12(1.94)$ & $6.05(1.80)$ & 0.33 \\
t†LH (IU/L) & $5.71(2.72)$ & $5.69(2.50)$ & $6.07(2.46)$ & 0.70 \\
\hline
\end{tabular}

*BMI: Basal metabolic index; ${ }^{* *} \mathrm{ET}$ : Endometrial thickness; ${ }^{* *} \mathrm{SD}$ : Standard deviation; $\dagger \mathrm{FSH}$ : Follicle-stimulating hormone; ††LH: Luteinizing hormone; $€ p<0.05$ : Statistically significant 
pregnancy rates (CPR) and implantation rates (IR) were significantly higher in women with zone 3 or 4 penetration [CPR 40.84\% (29/71) and IR 19.78\% (37/187) respectively] compared with zone 1 or 2 penetration [CPR $15.78 \%$ $(3 / 19)$ and $21.62 \%(8 / 37)$, IR 7.84\% (4/51) and 9\% (9/100) respectively, $\mathrm{p}=0.03$ and 0.01 , respectively] (Graph 1).

There was no difference in the semen parameters across the three groups.

\section{DISCUSSION}

Implantation is the result of a good quality embryo, a receptive endometrium and a cross-talk between the two. Endometrial receptivity is an enigma. Ultrasound enables a noninvasive way to characterize the endometrium. Furthermore, addition of Doppler allows assessment of endometrial vasculature which has been shown to play a prominent role in early endometrial response to the implanting blastocyst. Also, it has been suggested that vascular changes may contribute to uterine receptivity. ${ }^{11}$

Correlation between blood flow in subendometrialendometrial unit and the pregnancy rates in IVF cycles has been evaluated in several studies. Some workers have suggested positive correlation of pregnancy rates with the subendometrial-endometrial blood flow, ${ }^{3-5,12-16}$ while others have refuted this. ${ }^{17-22}$

Scoring systems have also been formed to predict pregnancy outcome using endometrial vascularization to depict the chances of pregnancy. ${ }^{10}$ According to Applebaum, ${ }^{10}$ most fertile patients usually demonstrate flow into zone 3 by the midcycle. Inadequate vascular penetration of the endometrium (not within zone 3) prior to transfer has been associated with an unfavorable outcome. These Doppler findings in unsuccessful cycles

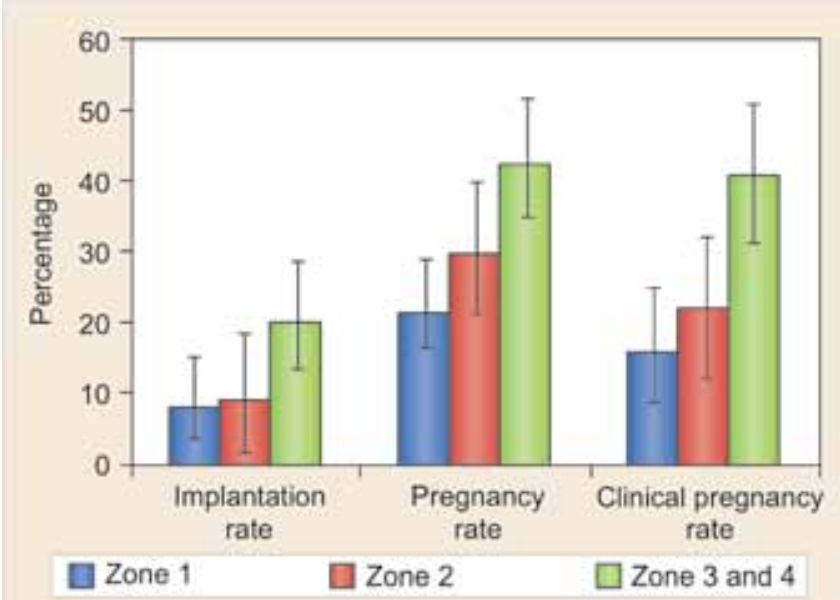

Graph 1: The above bar graph shows the comparison between the percentage of observed events Implantation, pregnancy and clinical pregnancy rates for patients with blood flow in zones 1,2 and 3 or 4 respectively. The error bars represent the $95 \%$ confidence intervals for the estimated proportions may relate to the histologic findings described by Sterzik et $\mathrm{al}^{23}$ where majority demonstrated an immature endometrium at the time of embryo transfer. The abnormalities included a variety of patterns, all indicating a lack of secretory transformation, reflecting unpreparedness for implantation.

Quantitative assessment of vessel density and perfusion within subendometrial area using 3D-PD was found to be the strongest predictive factor of IVF success among various sonographic parameters. ${ }^{15,24}$ Singh et al also stated that endometrial vascularity by PD has a useful predictive value for implantation rate in IVF cycles irrespective of morphological appearance of endometrium. ${ }^{16}$

Salleetalstated thatindividual sonographic and Doppler parameters are not of sufficient accuracy to predict uterine receptivity. However, the uterine score calculated prior to IVF-ET cycles appeared to be a useful predictor of implantation. ${ }^{25}$ But Yuval et al did not find any correlation between endometrial thickness, blood flow and pregnancy rate in IVF. ${ }^{26}$ De Ziegler and Focydman ${ }^{27}$ also stated that Doppler USG of endometrial and subendometrial regions does not contribute valid responses as to uterine receptivity and the chances for pregnancy.

Zaidi et $\mathrm{al}^{5}$ reported that absence of blood flow in endometrium and subendometrial zones was associated with failure of implantation ( $\mathrm{p}<0.05)$. Also, the pregnancy rates are related to the zones of vascular penetration into the subendometrial and endometrial regions (26.7\% for zone 1, 36.4\% for zone 2 and 37.9\% for zone 3), but were not significantly different.

Similarly, Chien et $\mathrm{al}^{4}$ found that pregnancy and implantation rates of patients with presence of both endometrial and subendometrial blood flow was 47.8 and $24.2 \%$ respectively, for patients with blood flow in the subendometrial unit only was 29.7 and $15.8 \%$ respectively, and for patients with no detectable endometrialsubendometrial blood flow was 7.5 and $3.5 \%$ respectively. They concluded that endometrial-subendometrial blood flow distribution pattern assessed by transvaginal Color Doppler before ET is correlated with implantation and pregnancy rates.

Wang et $\mathrm{al}^{28}$ divided patients undergoing IVF-ET into 3 groups: with undetectable endometrial blood flow, with subendometrial blood flow and with both endometrial and subendometrial blood flow. They found that patients with detectable endometrial blood flow had higher clinical pregnancy rates and implantation rates.

However, Baruffi et $\mathrm{al}^{29}$ in 2001 could not find evaluation of endometrial vascularization using PD as a predictor of pregnancy in an ICSI program. Similar results were reported by Aghahoseini et $\mathrm{al}^{30}$ in their study. 
Hence, there are conflicting reports on the role of endometrial vascularity in predicting pregnancy outcome in IVF cycles. There is a scarcity of data relating the same in FET cycles. Tekay et al found no difference in Doppler velocimetry measurements between the conception and non-conception cycles in both the FET or IVF-ET groups. They then concluded that an inadequate uterine blood flow impaired implantation, while optimum uterine blood perfusion did not necessarily lead to conception. ${ }^{7}$

Check et al and Zackova et al concluded that measurement of Power Doppler indices does not provide any additional information concerning the outcome of FET cycles. ${ }^{12,31}$ Ernest et al found that the vascularity of endometrium and subendometrial layers measured by 3D-PD USG was not a good predictor of pregnancy during stimulated IVF and FET cycles if it was measured at one time point only. ${ }^{32}$

In contrast, we found that with increased endometrial blood flow (subendometrial flow to outer hyperechoic region of endometrium to inner hypoechoic region of endometrium and hyperchogenic cavity), there was improvement in the pregnancy rate though the result was not statistically significant ( $p=0.15)$. But, it is seen that there was a rising trend in the pregnancy rate (PR) as the endometrial blood flow increases with a PR of $21.05 \%$ when the blood flow was seen up to zone 1 compared to PR of $42.25 \%$ in those with blood flow up to zone 3 or 4 showing a clinically significant improvement in the effect size with increasing endometrial blood flow. However, we found statistically significant increase in the clinical pregnancy rate with increasing endometrial blood flow $(p=0.03)$. The implantation rate also improved significantly with increased endometrial blood flow $(p=0.01)$. The observed differences in pregnancy rate, clinical pregnancy rate and implantation rate could be due to small study population.

To summarize, amount of endometrial blood flow correlates with pregnancy outcome in HRT-FET cycles.

The differences in observations could be due to differences in the technique of freezing used, developmental stage of embryos at the time of freezing, protocol used for endometrial preparation, endometrial thickness at the time of transfer or the technique of Doppler used for assessing endometrial blood flow.

Doppler analysis of endometrial blood flow is a simple and effective tool to improve clinical pregnancy rate in HRT-FET cycles and should be incorporated into routine clinical practice. Moreover, as implantation rate improves with increased endometrial blood flow, we can reduce the number of embryos to be transferred and hence, the multiple pregnancy rates in FET cycles with good endometrial blood flow. If we can improve pregnancy rates in
FET cycles, it can also help to reduce the risk of ovarian hyperstimulation syndrome (OHSS) by using GnRH agonist trigger and/or by elective cryopreservation of embryos in high risk cases. However, this analysis needs to be complemented by research correlating this study protocol with live birth rate.

One limitation of our study could be the small number of patients studied. Another limitation was significant difference in the age of women between the different zones. Possibly larger studies are required to reach definite conclusions.

\section{CONCLUSION}

Clinical pregnancy rate and implantation rate improves significantly with increased amount of endometrial blood flow on 2D-PD in HRT-FET cycles.

We suggest that a combination of endometrial thickness and Doppler analysis of endometrial blood flow is a simple and effective tool to improve clinical pregnancy rate in HRT-FET cycles and should be incorporated into routine clinical practice. Also, this can help us reduce the number of embryos to be transferred and hence, the multiple pregnancies and the associated complications.

\section{REFERENCES}

1. Friedler S, Schenker JG, Herman A, Lewin A. The role of ultrasonography in the evaluation of endometrial receptivity following assisted reproductive treatments: a critical review. Hum Reprod Update 1996;2(4):323-335.

2. Merce LT. Ultrasound markers of implantation. Ultrasound Rev Obstet Gynecol 2002;2:110-123.

3. Merce LT, Barco MJ, Bau S, Troyano J. Are endometrial parameters by three-dimensional ultrasound and power Doppler angiography related to in vitro fertilization/embryo transfer outcome? Fertil Steril 2008;89(1):111-117.

4. Chien LW, Au HK, Chen PL, Xiao J, Tzeng CR. Assessment of uterine receptivity by the endometrial-subendometrial blood flow distribution pattern in women undergoing in vitro fertilization-embryo transfer. Fertil Steril 2002;78(2):245-251.

5. Zaidi J, Campbell S, Pittrof R, Tan SL. Endometrial thickness, morphology, vascular penetration and velocimetry in predicting implantation in an in vitro fertilization program. Ultrasound Obstet Gynecol 1995;6(3):191-198.

6. Maugey-Laulom B, Commenges-Ducos M, Jullien V, Papaxanthos-Roche A, Scotet V, Commenges D. Endometrial vascularity and ongoing pregnancy after IVF. Eur J Obstet Gynecol Reprod Biol 2002;104(2):137-143.

7. Tekay A, Martikanien H, Jouppila P. Blood flow changes in uterine and ovarian vasculature and predictive value of transvaginal pulsed colour Doppler ultrasonography in an in vitro fertilization programme. Hum Reprod 1995;10(3): 688-693.

8. Bassil S, Margitte JP, Roth J, Nisolle M, Donnez J, Gordts S. Uterine vascularity during stimulation and its correlation with implantation and in vitro fertilization. Hum Reprod 1995;10(6):1497-1501. 
9. Kupesic S, Kurjak A. Uterine and ovarian perfusion during the periovulatory period assessed by transvaginal color Doppler. Fertil Steril 1993;60(3):439-443.

10. Applebaum M. The uterine biophysical profile. Ultrasound Obstet Gynecol 1995;5(1):67-68.

11. Rogers PAW. Structure and function of endometrial blood vessels. Hum Reprod Update 1996;2(1):57-62.

12. Check JH, Dietterich C, Lurie D, Choe JK, Nazari A. The relationship of color and power doppler ultrasound parameters of pulsatility and resistance indices and sub-endometrial blood flow with endometrial thickness on day prior to progesterone administration and their relationship to clinical pregnancy rate following frozen embryo transfer. Fertil Steril 2003;80(3):S123.

13. Bergh PA, Navot D. The impact of embryonic development and endometrial maturity on the timing of implantation. Fertil Steril 1992;58(3):537-542.

14. Garcia E, Bouchard P, De Brux J, Berdah J, Frydman R, Schaison $\mathrm{G}$, et al. Use of immunocytochemistry of progesterone and estrogen receptors for endometrial dating. J Clin Endocrinol Metab 1988;67(1):80-87.

15. Bergeron C, Ferenczy A, Toft DO, Schneider W, Shyamala G. Immunocytochemical study of progesterone receptors in the human endometrium during the menstrual cycle. Lab Invest 1988;59(6):862-869.

16. le Nestour E, Marraoui J, Lahlou N, Roger M, de Ziegler D, Bouchard P. Role of estradiol in the rise in follicle-stimulating hormone levels during the luteal-follicular transition. J Clin Endocrinol Metab 1993;77(2):439-442.

17. Navot D, Bergh PA, Williams M, Garrisi GJ, Guzman I, Sandler B, et al. An insight into early reproductive processes through the in vivo model of ovum donation. J Clin Endocrinol Metab 1991;72(2):408-414.

18. Navot D, Bergh P. Preparation of the human endometrium for implantation. Ann NY Acad Sci 1991;622:212-219.

19. Navot D, Anderson TL, Droesch K, Scott RT, Kreiner D, Rosenwaks Z. Hormonal manipulation of endometrial maturation. J Clin Endocrinol Metab 1989;68(4):801-807.

20. Swierz L, Giudence L. Unexplained infertility and the role of uterine receptivity. Clinics North America 1997;8:523-543.

21. Tur-kaspa I, Confino E, Dudkiewicz AB, Myers SA, Friberg J, Gleicher N. Ovarian stimulation protocol for in vitro fertilization with gonadotropin-releasing hormone agonist widens the implantation window. Fertil Steril 1990;53(5):859-866.
22. Fanchin R. Assessing uterine receptivity in 2001: ultrasonographic glances at the new millennium. Ann NY Acad Sci 2001;943:185-202.

23. Sterzik K, Dallenbach C, Schneider V, Sasse V, DallenbachHellweg G. In vitro fertilization: the degree of endometrial insufficiency varies with the type of ovarian stimulation. Fertil Steril 1988;50(3):457-462.

24. Navot D, Scott R, Droes K, Veeck L, Liu H, Rosenwak S. The window of embryo transfer and efficiency of human conception in vivo. Fertil Sertil 1991;55(1):114-118.

25. Salle B, Bied-Damon V, Benchaib M, Desperes S, Gaucherand P, Rudigoz RC. Preliminary report of an ultrasonography and colour Doppler uterine score to predict uterine receptivity in an in vitro fertilization programme. Hum Reprod 1998;13(6): 1669-1673.

26. Yuval Y, Lipitz S, Dor J, Achiron R. The relationships between endometrial thickness, and blood flow and pregnancy rates in in vitro fertilization. Hum Reprod 1999;14(4):1067-1071.

27. De Ziegler D, Frydman R. Different implantation rates after transfers of cryopreserved embryos originating from donated oocytes or from regular in vitro fertilization. Fertil Steril 1990; 54(4):682-688.

28. Wang L, Qiao J, Li R, Zhen X, Liu Z. Role of endometrial blood flow assessment with color Doppler energy in predicting pregnancy outcome of IVF-ET cycles. Reprod Biol Endocrinol 2010;18(8):122.

29. Baruffi RL, Contart P, Mauri AL, Peterson C, Felipe V, Franco JG. A uterine ultrasonographic scoring system as a method for the prognosis of embryo implantation in an ICSI program. Fertil Steril 2001;76(3):S189.

30. Aghahoseini M, Tuba K, Marsousi V, Aleyasin. Assessment of endometrial-subendometrial blood flow detected by color Doppler sonography and uterine receptivity in infertile women. Acta Medica Iranica 2008;46(6):461-466.

31. Zácková T, Järvelä IY, Tapanainen JS, Feyereisl J. Assessment of endometrial and ovarian characteristics using three dimensional power Doppler ultrasound to predict response in frozen embryo transfer cycles. Reprod Biol Endocrinol 2009;25(7):151.

32. Ng EHY, Chan CCW, Tang OS, Yeung WSB, Ho PC. The role of endometrial and subendometrial vascularity measured by three-dimensional power Doppler ultrasound in the prediction of pregnancy during frozen-thawed embryo transfer cycles. Hum Reprod 2006;21(6):1612-1617. 